\title{
Concrescence of Erupted Second Molar and Impacted Third Molar: A Rare Case Report
}

\author{
Dr. Vishal Tapadiya, Dr. Shandilya Ramanojam, Dr. Kalyani Gelada, \\ Dr. Sanjana Sethi, Dr. Nitin Oswal \\ (Department of Oral and maxillofacial Surgery, Bharati Vidyapeeth Dental College and Hospital, India, Pune)
}

\begin{abstract}
Concrescence is union of two adjacent teeth by cementum alone without confluence of the underlying dentin. The incidence of concrescence teeth is reported to be highest in the posterior maxilla. The presence of concrescence teeth may influence teeth extraction as well as periodontal, endodontic, orthodontic and even prosthodontic diagnosis and treatment planning. Unexpected complications arising from this condition may lead to legal complications. Therefore, consideration should be given to the possible occurrence, recognition, and implications of this anomaly in diagnosis and treatment planning. The rationale of this article is to report a case of teeth concrescence between an impacted third molar and an erupted second molar with that was identified post-extraction, with a review of literature

Keywords: concrescence, impacted third molar
\end{abstract}

\section{Introduction}

Concrescence of teeth is actually a form of fusion that occurs during root formation or after the radicular phase of development is complete. If concrescence occurs during development it is called as true concrescence and if occurs after development it is called acquired concrescence ${ }^{1}$. In order for concrescence to take place, the roots of the affected teeth must be in close proximity to each other, and an excess layer of cementum must be deposited to form the union between the roots of the adjacent teeth. Therefore, the union is only in the cementum of the adjacent teeth. One case was reported showing concrescence of the crown of an impacted tooth and the roots of the erupted tooth ${ }^{2}$. The degree of union may vary from one small site to a solid cemental mass along the entire extent of the root. Two adjacent roots become fused by deposition of cementum between them after the resorption of interdental bone. The process is noted more frequently in the posterior and maxillary region ${ }^{4}$. The developmental pattern often involves a second molar tooth, in which its roots closely approximate the adjacent impacted third molar. Its prevalence is not influenced by age, gender, or race. In this case the roots of third molar are located perpendicular to the root area of second molar. This type of concrescence is not reported before.

\section{Report of a Case}

A 35-year-old female patient presented at Bharati Vidyapeeth Dental College and Hospital, Pune (INDIA) with a complaint of pain in the upper right back teeth region lasted for three days. She mentioned history of dull continuous pain which aggravated on mastication and relieved temporarily on medication. The patient reported her past medical history to be negative. No history of any drug or food allergy. A clinical examination was performed. The examination identified few teeth to be non restorable due to caries or periodontal disease. No unusual pathological conditions were noted. Maxillary right second molar tender on percussion along with caries with pulpal involvement on the distal aspect as tooth was supposed to be saved by performing Root canal treatment. Maxillary right third molar is partially impacted as one cusp was visible. It was confirmed by radiographic investigation. It was planned to extract the third molar by raising the flap. With proper sterilization maxillary right third molar was extracted with slow luxation and bimanual palpation of the alveolar ridge. The upper right third molar was extracted without fracture of the tuberosity and with the second molar still fused to it. Both teeth were extracted through the site occupied by maxillary right third molar without tearing of the alveolar mucosa. The patient was explained about the situation and was prescribed medications for five days and combination of Diclofenac Sodium-50mg and Paracetamol-500mg twice daily for three days. The teeth were cleaned under running water and tissue tags were removed using a curette and preserved in a plastic bottle containing 3\% Weight/Volume hydrogen peroxide. Photographs were taken in different views (Figure $1,2)$ and radiograph using digital radiograph device to determine what odontogenic tissues were involved in the affected teeth(Figure 3). 


\section{FIGURES}

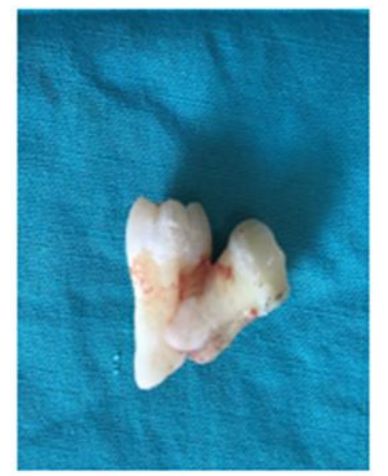

Figure-1

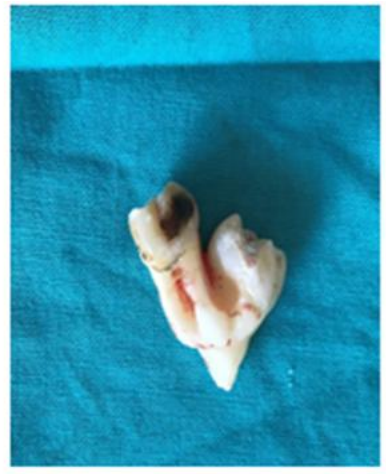

Figure-2

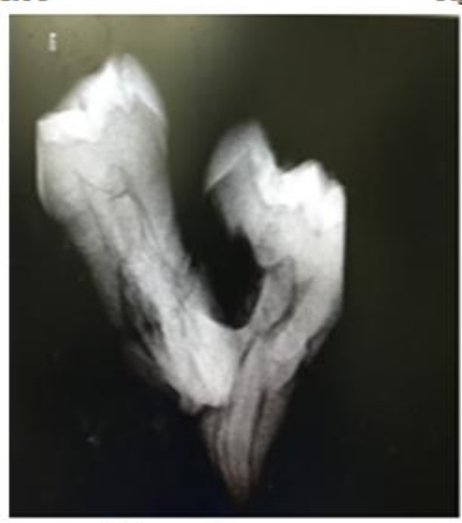

Figure-3

\section{Discussion}

Double teeth are two separate teeth exhibiting union by dentin and their pulps. The union may be the result of fusion of two adjacent tooth buds or the partially splitting of one into two. The development of isolated large or joined (i.e. double) teeth is not rare, but the literature is confusing when appropriate terminology is presented. Historically, germination is defined as an attempt of a single tooth bud to divide, with the resultant formation of a tooth with a bifid crown and usually a common root and a root canal. Conversely, fusion was considered the union of two normally separated tooth bud with the resultant formation of a joined tooth with confluence of dentin. Finally, concrescence represents an uncommon developmental anomaly in which juxtaposed teeth are united in the cementum but not in the dentin ${ }^{1}$.

Concrescence is believed to occur during root formation or after the radicular phase of development is complete. If concrescence occurs during root formation, it is categorized as developmental (true) and attributed to the close proximity of the developing roots of the adjacent teeth ${ }^{2}$. It occurs in apposition and maturation stages $^{3}$. If concrescence occurs after root formation, it is categorized as post-inflammatory (acquired) and it may result from a chronic inflammatory response to a non-vital tooth. Although, its cause is unknown it is suspected that space restriction during development, local trauma, excessive occlusal forces or local infection after development plays an important role ${ }^{4}$. The detection of concrescence is important because of the potential complication it poses during extraction and endodontic therapy. It is impossible to be detected clinically, and may defy radiographic detection as well when it may be misdiagnosed as simple radiographic overlap or superimposition of adjacent teeth. Therefore, it is important to consider this possibility when the roots of adjacent teeth are radiographically indistinguishable. Radiographs with different angulations and exposure parameters may aid in diagnosis. Concrescence teeth may give rise to complications, such as an extraction of an adjacent tooth, fracture of the tuberosity or floor of the maxillary sinus ${ }^{5}$. Therefore, it is very important to inform the patient about the condition and potential complications. In such cases, sectioning should be considered to minimize adverse and unexpected outcomes.

\section{Conclusion}

Diagnosis of teeth concrescence occurs mainly after a surgical mishap. Therefore, it is important for clinicians to be aware of such developmental tooth anomaly in order to minimize undesirable and unpredicted outcomes during dental treatment. 


\section{References}

[1]. Denise Foran et al; Concrescence of permanent maxillary second and third molars: case report of non-surgical root canal treatment; Journal of Oral Science, Vol. 54, No. 1, 133-136, 2012

[2]. V Bellapu, S Nagarakanti; Concrescence of Erupted Second Molar and Impacted Third Molar: A Rare Case Report. The Internet Journal of Dental Science. 2009 Volume 8 Number 2.

[3]. Jan 5, 2015, Mrzezo General Dentistry, MJ Fehrenbach 2011, seatle WA

[4]. William K. Duncan et al; Bilateral fusion and gemination: A literature analysis and case report; ORAL SURC. ORAL MED. ORAL PATHOL. 1987; 64:82-7)

[5]. G S Kumar; Orban's Oral histology and embryology; Elsevier $12^{\text {th }}$ Edition

[6]. R. Rajendran; Shafer's Textbook of Oral Pathology; Elsevier $6^{\text {th }}$ Edition. 